\title{
Preoperative localization of adult nesidioblastosis using ${ }^{68}$ Ga-DOTA-exendin-4-PET/CT
}

\author{
Emanuel Christ $^{1}$ - Damian Wild ${ }^{2}$ Kwadwo Antwi ${ }^{2} \cdot$ Beatrice Waser $^{3}$. \\ Melpomeni Fani $^{2} \cdot$ Stefanie Schwanda $^{4} \cdot$ Tobias Heye $^{2} \cdot$ Christoph Schmid $^{5}$. \\ Hans Ulrich Baer ${ }^{6} \cdot$ Aurel Perren $^{3} \cdot$ Jean Claude Reubi $^{3}$
}

Received: 18 April 2015/Accepted: 13 May 2015/Published online: 23 May 2015

(C) Springer Science+Business Media New York 2015

The term "nesidioblastosis" has been used to denote histopathological findings such as neogenesis from pancreatic ductal epithelium in parallel with $\beta$-cell hyperplasia and hypertrophy (1). This entity was first described in children and neonates and is characterized by endogenous hyperinsulinemic hypoglycemia. The incidence rate in adults ranges between 0.5 and $7 \%$. The pathophysiological mechanisms in adults are ill-defined.

The preoperative distinction between insulinoma and nesidioblastosis is of primordial importance since the therapeutical strategies are different, ranging from a enucleation in the case of insulinoma up to a $70-80 \%$ pancreatectomy in the case of nesidioblastosis. Clinically, the distinction between insulinoma and nesidioblastosis is not always possible.

Emanuel Christ and Damian Wild are co-first authors.

Jean Claude Reubi

jean.reubi@pathology.unibe.ch

1 Department of Endocrinology, Diabetology and Clinical Nutrition, University Hospital of Bern, Inselspital, Bern, Switzerland

2 Clinic of Radiology and Nuclear Medicine, University of Basel Hospital, Basel, Switzerland

3 Division of Experimental Pathology, Department of Pathology, Institute of Pathology, University of Bern, 3010 Bern, Switzerland

4 Department of Internal Medicine, Limmattalspital Schlieren, Schlieren, Switzerland

5 Division of Endocrinology, Diabetology and Clinical Nutrition, University Hospital of Zürich, Zurich, Switzerland

6 Department of Visceral Surgery, Hirslanden Clinic, Zurich, Switzerland
Preoperative imaging including MRI, CT, and endosonography is not diagnostic in adult nesidioblastosis, and invasive investigation using selective arterial calcium stimulation and venous sampling (ASVS) is recommended. Although this investigation is often helpful, it is an invasive procedure with the associated risks. Recently, we have shown that targeting glucagon-like peptide-1 receptor (GLP-1R) using ${ }^{111}$ In-DOTA-exendin-4 SPECT/CT is a very sensitive non-invasive method to localize benign insulinoma (2). Whether GLP-1R targeting is useful in adult nesidioblastosis is currently unknown.

\section{Case report}

In a 65-year-old woman with hypoglycemic symptoms, fasting endogenous hyperinsulinemic hypoglycemia was confirmed. Abdominal MRI, endosonographies, ${ }^{18} \mathrm{~F}$ DOPA-PET/CT, ${ }^{68} \mathrm{Ga}$-DOTATATE-PET/CT, and ASVS were negative or inconclusive.

She was included in a prospective study that compares ${ }^{111}$ In-DOTA-exendin-4 SPECT/CT with ${ }^{68}$ Ga-DOTA-exendin-4 PET/CT (ClinicalTrials.gov, No. NCT02127541). ${ }^{111}$ In DOTA-exendin-4, SPECT/CT was not conclusive, but ${ }^{68} \mathrm{Ga}$-DOTA-exendin-4 PET/CT documented an increased diffuse uptake from the tail of the pancreas to the pancreatic corpus $\left(\mathrm{SUV}_{\max } 6.9\right.$ Fig. 1a).

A left-sided pancreatectomy was performed. In vitro detection of GLP-1Rs using autoradiography revealed a more than 3-time increase in the number of GLP-1Rs in the nesidioblastosis islets in all four samples (density $($ mean $\pm \mathrm{SEM}): \quad 3606 \pm 340 \mathrm{dpm} / \mathrm{mg}$ tissue; Fig. $1 \mathrm{~b}$ ) versus normal islets (3). Conversely, GLP-1R density in the exocrine acini of the pancreas with nesidioblastosis was not increased, as compared to normal acini. Histological 

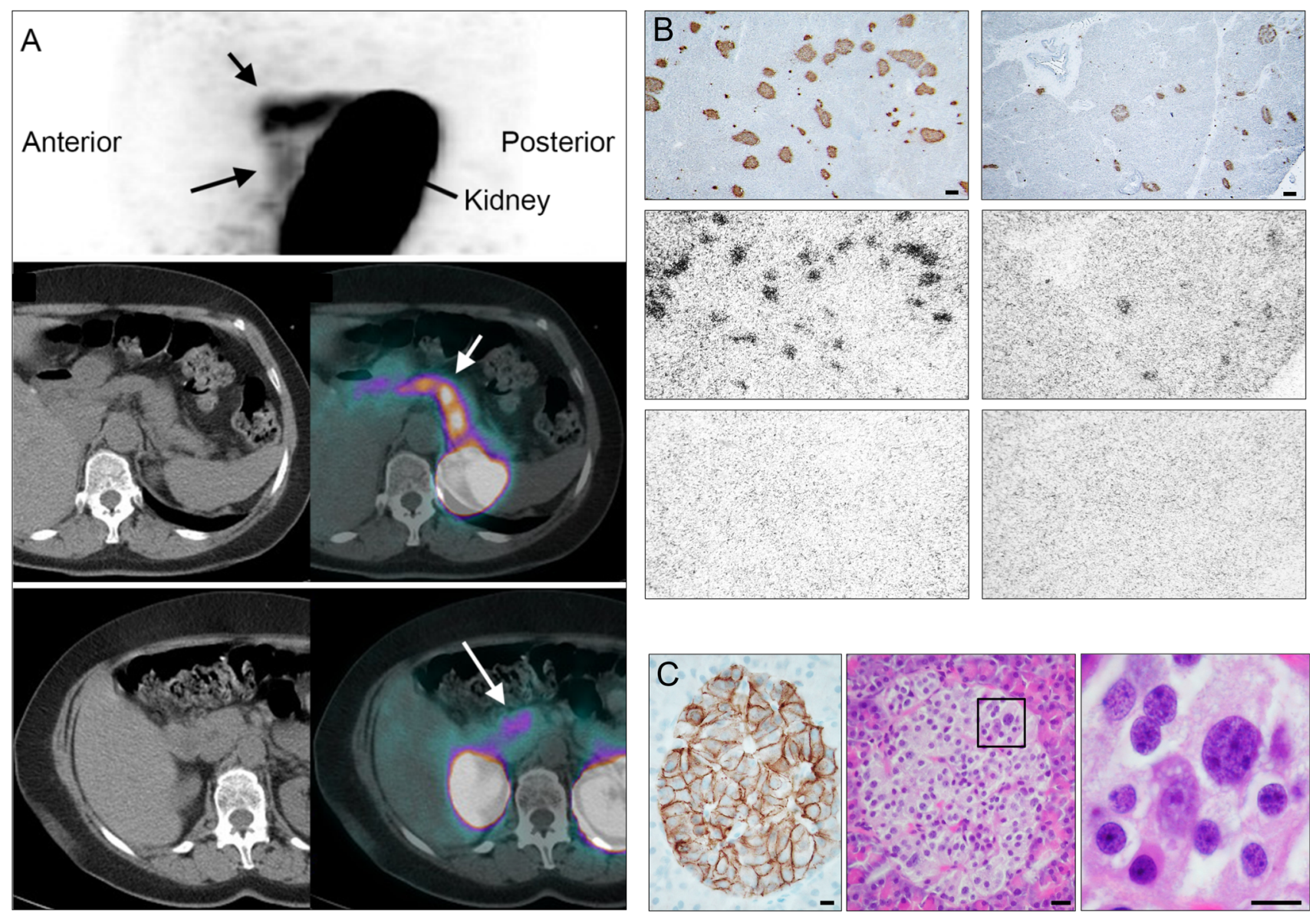

Fig. 1 a Sagittal view of ${ }^{68} \mathrm{Ga}$-DOTA-exendin-4 abdominal PET scan (top), trans-axial view of ${ }^{68} \mathrm{Ga}$-DOTA-exendin-4 PET/CT, and corresponding CT scan (middle at the level of pancreatic corpus/tail, bottom at the level of pancreatic head). The pancreatic corpus and tail are highly ${ }^{68} \mathrm{Ga}$-DOTA-exendin-4 avid (small arrows), whereas the pancreatic head shows only little ${ }^{68}$ Ga-DOTA-exendin-4 uptake (large arrows). b In vitro GLP-1R autoradiography of the pancreas from the nesidioblastosis case (left column) and normal pancreas (right column). First row is insulin immunostaining showing an increased number and enlarged islets in the nesidioblastosis compared to few islets in normal tissue. Second row are autoradiograms

specimen confirmed nesidioblastosis, and GLP-1R immunohistochemistry revealed GLP-1Rs localized on the membrane of insulin-producing cells in the islets of the nesidioblastosis patient (Fig. 1c).

Postoperatively, the hypoglycemia resolved. The main messages of this case report are as follows: (1) Adult nesidioblastosis can non-invasively be suspected using ${ }^{68} \mathrm{Ga}-$ DOTA-exendin-4 PET/CT. (2) The molecular rationale for it is found in the present autoradiography studies that demonstrate a more than 3-time higher density of GLP-1R in the islets of this nesidioblastosis patient, when compared with islets of normal pancreas. The immunohistochemical findings indicate that these GLP-1R are predominantly expressed by the insulin-producing islet cells. The

showing total binding of ${ }^{125}$ I-GLP-1(7-36) amide. The islets in the nesidioblastosis are more strongly labeled than in the normal pancreas. Third row is non-specific binding. Bars $1 \mathrm{~mm}$. c Left GLP-1R immunohistochemistry in an islet from the nesidioblastosis case. Strong membranous staining. Middle and right hematoxylineosin-stained section showing an islet of the presented case with single large beta cells with hyperchromatic nuclei, some with enlarged nuclei, clear halo around beta cells, and a discrete lobulated structure, histopathologically typical for nesidioblastosis. Bars $0.01 \mathrm{~mm}$

increased number of GLP-1R in $\beta$-cells is further potentiated by a considerable increase in number and size of the islets in this nesidioblastosis case. (3) The amount of GLP$1 \mathrm{R}$ in the nesidioblastosis pancreas is higher than in a normal pancreas, but lower than in benign insulinomas, reflecting the moderate intensity of the in vivo scan in this patient. (4) Since adult nesidioblastosis can be a focal disease, a non-invasive preoperative tool like ${ }^{68} \mathrm{Ga}$-DOTAexendin-4 PET may be helpful in determining the surgical strategy.

Acknowledgements We thank Thomas Pfammatter, University Hospital of Zurich, for performing ASVS testing and Daniela Biondo, Mia Nagy, Svenja Scheiwiller, Franziska Hofer, and Andrea Popescu (University of Basel Hospital) for excellent technical assistance in 
carrying out GLP-1R imaging. This work was supported in part by the Swiss National Science Foundation Grant No. 320030_152938/1 (to E.C., D.W. and M.F.) and the Desirée and Niels Yde's Foundation Grant No, 389-12 (to D.W.).

Conflict of interest The authors declare that they have no conflict of interest.

\section{References}

1. M. Anlauf, D. Wieben, A. Perren, B. Sipos, P. Komminoth, A. Raffel, M.L. Kruse, C. Fottner, W.T. Knoefel, H. Monig, P.U.
Heitz, G. Kloppel, Persistent hyperinsulinemic hypoglycemia in 15 adults with diffuse nesidioblastosis: diagnostic criteria, incidence, and characterization of beta-cell changes. Am. J. Surg. Pathol. 29, 524-533 (2005)

2. E. Christ, D. Wild, S. Ederer, M. Béhé, G. Nicolas, M. Caplin, M. Brändle, T. Clerici, S. Fischli, C. Stettler, P. Ell, J. Seufert, B. Gloor, A. Perren, J.C. Reubi, F. Forrer, Glucagon-like peptide-1 receptor imaging for the localisation of insulinomas: a prospective multicentre imaging study. Lancet Diabetes Endocrinol 1, 115-122 (2013)

3. M. Korner, M. Stockli, B. Waser, J.C. Reubi, GLP-1 receptor expression in human tumors and human normal tissues: potential for in vivo targeting. J. Nucl. Med. 48, 736-743 (2007) 